\title{
Physical activity and hypocaloric diet recovers osteoblasts homeostasis in women affected by abdominal obesity
}

\author{
Viviana M. Bimonte ${ }^{1} \cdot$ Simona Fittipaldi ${ }^{1,2} \cdot$ Chiara $^{\text {Marocco }}{ }^{3}$. \\ Gian Pietro Emerenziani ${ }^{1}$ - Rachele Fornari ${ }^{3}$ - Laura Guidetti ${ }^{1}$. \\ Eleonora Poggiogalle ${ }^{3}$ Emanuele Nicolai ${ }^{2}$. Luigi Di Luigi ${ }^{1}$ Lorenzo M. Donini ${ }^{3}$. \\ Carlo Baldari ${ }^{1} \cdot$ Andrea Lenzi $^{3} \cdot$ Emanuela A. Greco $^{3} \cdot$ Silvia Migliaccio $^{1}{ }^{1}$
}

Received: 17 July 2016 / Accepted: 28 November 2016

(C) Springer Science+Business Media New York 2016

\begin{abstract}
Obesity is a multifactorial disease linked to metabolic chronic disorders such as diabetes, and hypertension. Also, it has recently been associated with skeletal alterations and low bone mineral density. We previously demonstrated that exposure of osteoblasts to sera of sedentary subjects affected by obesity alters cell homeostasis in vitro, leading to disruption of intracellular differentiation pathways and cellular activity. Thus, the purpose of the present study has been to evaluate whether sera of sedentary obese women, subjected to physical activity and hypocaloric diet, could recover osteoblast homeostasis in vitro as compared to the sera of same patients before intervention protocol. To this aim, obese women were evaluated at time 0 and after 4,6 , and 12 months of individualized prescribed physical activity and hypocaloric diet. Dual-energy-X-ray absorptiometry measurements were performed at each time point, as well as blood was collected at the same points. Cells were incubated with sera of subjects before and after physical activity as described: obese at baseline and after for 4, 6, and 12 months of physical activity and nutritional protocol intervention. Osteoblasts exposed to sera of patients, who displayed increased lean and decreased fat mass (from $55.5 \pm 6.5$ to $57.1 \pm 5.6 \% p \leq 0.05$; from $44.5 \pm 1.1$ to
\end{abstract}

Silvia Migliaccio

silvia.migliaccio@uniroma4.it

1 Department of Movement, Human and Health Sciences, Section of Health Sciences, Foro Italico University, Rome, Italy

2 IRCCS S.D.N., Naples, Italy

3 Department of Experimental Medicine, Section of Medical Pathophysiology, Endocrinology and Nutrition, Sapienza University, Rome, Italy
$40.9 \pm 2.6 \% \quad p \leq 0.01$ respectively), showed a timedependent increase of $\mathrm{Wnt} / \beta$-catenin signaling, versus cells exposed to sera of obese patients before intervention protocol, suggesting recovery of osteoblast homeostasis upon improvement of body composition. An increase in $\beta$-catenin nuclear accumulation and nuclear translocation was also observed, accompanied by an increase in Adiponectin receptor 1 protein expression, suggesting positive effect on cell differentiation program. Furthermore, a decrease in sclerostin amount and an increase of type 1 procollagen amino-terminal-propeptide were depicted as compared to baseline, proportionally to the time of physical activity, suggesting a recovery of bone remodeling modulation and an increase of osteoblast activity induced by improvement of body composition. In conclusion, our results show for the first time that sera of obese sedentary women who increased lean mass and decreased fat mass, by physical activity and hypocaloric diet, rescue osteoblasts differentiation and activity likely due to a reactivation of Wnt/ $\beta$-catenin-pathway, suggesting that a correct life style can improve skeletal metabolic alteration induced by obesity.

Keywords Obesity $\cdot$ Visceral adipose tissue $\cdot$ Osteoblast homeostasis $\cdot$ Physical activity

\section{Introduction}

Obesity and osteoporosis are pandemic health problems with high prevalence and impact on mortality, morbidity, disability, and quality of life in affected individuals [1,2]. During the last decades, both diseases have become a major health threat around the world, with age and female status 
increasing the risk of developing both obesity and osteoporosis $[1,2]$.

Interestingly, obesity has been considered a risk factor for several metabolic chronic diseases (i.e. cardiovascular disease, diabetes mellitus) but a protection factor against the development of bone loss and osteoporosis, likely for both an increased androgen aromatization to estrogens in postmenopausal obese women [3, 4] and a potential role of mechanical loading in regulating bone remodeling [5]. Recently, however, the belief that obesity is protective against osteoporosis has been questioned. In fact, epidemiologic and clinical studies suggest that high level of abdominal fat mass might be a risk factor for a loss of bone density and/or quality leading to osteoporosis and fragility fractures [6-8]. Adipose tissue functions as an endocrine organ by releasing several adipokines, which seem to modulate glucose and lipid metabolism, inflammation and insulin resistance [9-11]. A pathological implication of adipose tissue for skeletal health could possible resides in the role that pro-inflammatory cytokines might play by interfering with bone remodeling [12-15]. More recently, obesity has also been associated to sarcopenia, a condition characterized by progressive decline of muscle mass, quality, and strength $[16,17]$. Further, as above mentioned, obesity is associated with a chronic low-grade inflammation as depicted by increased plasma levels of C-reactive protein, pro-inflammatory cytokines, and osteopontin $[18,19]$ as well as lower circulating levels of Vitamin D (Vit D) [20]. Thus, the interplay between fat and bone might play an important role as homeostatic feedback system in which adipokines and molecules secreted by bone or adipose cells represent the link of an active and functional bone-adiposemuscle axis [21-24], by mechanism(s) not fully clarified yet.

Remarkably, our group recently demonstrated that exposure of osteoblasts in vitro to the sera of sedentary obese women, affected by abdominal fat tissue accumulation, significantly disrupted osteoblast homeostasis by a Wnt/ $\beta$-catenin-dependent mechanism [25].

Thus, aim of the present study was to investigate whether serum factors in sedentary obese women who underwent an improvement of body composition, due to physical activity protocol and hypocaloric diet, could positively influence osteoblastic cells activity and differentiation recovering bone cells homeostasis in vitro as compared to the serum of the same subjects before intervention protocol.

\section{Materials and methods}

\section{Subjects}

Twenty-eight adult female (mean age $53 \pm 8.2$ years) affected by obesity (BMI $\geq 30 \mathrm{~kg} / \mathrm{m}^{2}$ ) were selected in the
Department of Experimental Medicine, Section of Medical Pathophysiology, Endocrinology, and Nutrition, of Policlinico Umberto I, Sapienza University of Rome. Twenty women were in menopause and eight women were in climacteric period, diagnosed by low estrogen levels and alterations of menstrual cycle. Chronic medical conditions (i.e. reumatological, gastroenterological pathologies, etc), use of medications affecting bone metabolism, hormonal and nutritional status, Vitamin D supplementation, recent weight loss, and prior bariatric surgery interventions were considered as exclusion criteria. Four subjects were diabetic, but they took antidiabetic oral medication, which did not affect mineral or inflammatory status. All subjects provided a written informed consent and the study was approved by the Local Ethical Committee. All subjects were sedentary and they had not been previously engaged in regular physical exercise. Subsequently, patients underwent an adequate physical activity program in combination with a hypocaloric equilibrate diet. After 4, 6, and 12 months patients were characterized for their body composition. The sera of obese women that showed, after physical activity and diet, an increased muscle mass and decrease abdominal fat tissue were used to culture osteoblasts and perform in vitro experiments.

\section{Nutritional evaluation}

Dietary counseling was performed by a nutritionist and a hypocaloric diet was set at approximately $400 \mathrm{Kcal}$ less than total daily energy expenditure. Total daily energy expenditure was determined by considering the resting metabolic rate and the physical activity level [26]. Physical activity level was estimated by the international physical activity questionnaire [27].

\section{Physical activity program}

The obese women enrolled in the study performed an unsupervised 12-months daily aerobic training based on a walking speed and on the value of RPE-OMNI-Walk/Run scale corresponding to their individual ventilatory threshold $[28,29]$. The training session duration varied lasting $30 \mathrm{~min}$ the first 2 months and 60 min the following 10 months. Each subject filled a physical activity diary day-by-day over the period of training. Every 2 months, subject's gait speed and RPE-OMNI-Walk/Run scale value, corresponding to individual ventilator threshold, were revaluated in order to prescribe a new work load for the following training period in order to maintain the individual aerobic exercise intensity constant during the 12 months. 


\section{Body composition}

Each subject's height was measured using a stadiometer to the nearest $0.1 \mathrm{~cm}$. Percentage of fat mass (\%FM), muscle mass in kilograms (FFM), bone density and body mass index (BMI) were assessed by dual-energy-X-ray absorptiometry (DXA, Hologic 4500 RDR). Amount of trunk fat mass was distinguished from peripheral and appendicular fat mass as a measure of abdominal adiposity by DXA with coefficient of variation of $<1 \%$ for bone density and $<1.5 \%$ for fat mass [7]. In particular, trunk fat was defined as the adipose tissue localized within the region below the chin, delineated by vertical lines within the left and right glenoid fossae bordering laterally to the ribs, and by the oblique lines that cross the femoral necks and converge below the pubic symphysis.

\section{Cell culture}

Human SAOS-2 osteoblastic cells were cultured in Dulbecco's modified Eagle's medium without Phenol Red, supplemented with $2 \mathrm{mM}$ L-glutamine, $100 \mathrm{U} / \mathrm{mL}$ penicillin/ streptomycin, $10 \%$ fetal bovine serum, at $37^{\circ} \mathrm{C}$ and $5 \%$ $\mathrm{CO}_{2}$ in a humidified incubator. Before starting each experiment, a pool from five different patients sera from the same time group was prepared and used for cellular treatments. Cells were grown up to $70 \%$ confluence, starved overnight in a medium serum-free, and then incubated $48 \mathrm{~h}$ with medium supplemented with $20 \%$ patients sera pools [25]. All buffers, media, and reagents were purchased from Euroclone (Milan, Italy). Cells were incubated with sera of subjects before and after physical activity as described: (1) obese (T0), (2) obese after for 4 months (T4), (3) obese after 6 months (T6), (4) obese after for 12 months of physical activity and nutritional protocol intervention (T12).

\section{Protein extraction and western blot analysis}

Following experimental treatments, cells were washed twice with $1 \times$ PBS, collected with scraper, and to extract total proteins, lysed in ice-cold RIPA buffer supplemented with protease and phosphatase inhibitor cocktails (SigmaAldrich, St. Louis, MO, USA).

For protein fraction analysis, lysates were precleared by centrifugation and supernatant (total proteins) was collected and stored at $-80{ }^{\circ} \mathrm{C}$ until use. NE-PER nuclear and cytoplasmic extraction Reagents (Thermo Fisher Scientific, Waltham, MA, USA) were used to extract nuclear and cytosolic fraction of cell proteins, following manufacturer's protocol. Protein amount was measured by using the Pierce $₫$ BCA protein assay kit (Thermo Fisher Scientific). An equal amount of protein extracts $(20 \mu \mathrm{g})$ were resolved in SDS-polyacrylamide gels (10-14\%) and transferred to nitrocellulose membranes (Amersham Biosciences, Pittsburgh, PA, USA). Membranes were saturated by incubation at RT for $1 \mathrm{~h}$ with $5 \%(\mathrm{w} / \mathrm{v})$ non-fat dry milk in $20 \mathrm{mM}$ Tris- $\mathrm{HCl}, 150 \mathrm{mM} \mathrm{NaCl}, 0.05 \%$ Tween 20 and incubated with specific antibodies overnight at $4{ }^{\circ} \mathrm{C}$.

Antibodies against $\beta$-catenin, tubulin and histone $\mathrm{H} 3$ were purchased from Abcam (Cambridge, UK), anti-TCF1, anti cMyc, anti c-Jun, and anti-CD44 antibody were purchased from Cell Signaling (Danvers, MA, USA). Cyclin D1 and adiponectin receptor-1 (AdipoR1) were purchased from Santa Cruz Biotechnology (Santa Cruz, CA, USA). All primary antibodies were diluted 1:1000 in 1× TBST with 5\% BSA or $5 \%$ non fat dry milk and incubated over night at $+4{ }^{\circ} \mathrm{C}$.

The immunoreactive protein bands were detected by incubation for $1 \mathrm{~h}$ with horseradish peroxidase-conjugated secondary goat anti-rabbit or goat anti-mouse (Jackson Laboratories, Bar Harbor, ME, USA, 1:10000) in blocking solution at room temperature, and they were visualized by enhanced chemiluminescence (Amersham Biosciences). The bands were acquired on the ImageQuant LAS 4000 (GE Healthcare) and quantified by ImageJ software.

\section{Elisa assay}

Osteoblast conditioned supernatants were collected and analyzed for Sclerostin (MyBioSource, San Diego, USA) and type 1 procollagen amino-terminal-propeptide (P1NP) (Cusabio, Hubei Province, China) content using commercially available ELISA kits according to the manufacturer's protocols.

Each experiment was performed in duplicate and repeated three times. Protein secretion levels found in the supernatant were corrected per mg of cellular protein of each dish. Total protein concentrations were determined with the BCA methods. Data are expressed as pg/mg protein.

\section{Statistical analysis}

Results are expressed as means $\pm \mathrm{SD}$ of at least three independent experiments. The significance of differences among results was evaluated using one-way ANOVA followed by Bonferroni post-hoc test (GraphPad Prism, San Diego, CA, USA). Data with $p<0.05$ was considered significant.

\section{Results}

\section{Patient characteristics}

Baseline characteristics of obese women enrolled in the study were: age $53 \pm 8.2$, body weight: $101.3 \pm 3.9 \mathrm{~kg}$, BMI $37.1 \pm 6.0 \mathrm{~kg} / \mathrm{m}^{2}$. These sedentary obese women underwent 
specific and individualized hypocaloric diet and physical activity protocol and significantly improved their body composition, as shown in Table 1 , with a decrease of total body weight from $101.3 \pm 3.9$ at baseline to $91.0 \pm 9.5^{*} \mathrm{~kg}$ after 12 months of the intervention protocol. Decrease of body weight in obese women was due to a loss of both fat and fat free mass (Table 1). However, when results were carefully evaluated it was possible to note that there was a significant decrease in the percentage of fat mass and a significant increase of the percentage of fat free mass, indicating a significant improvement of body composition in term of decrease of fat tissue (Table 1). Body composition variation was achieved after 4 months and maintained for all the intervention periods (Table 1). Due to the relative young age of subjects, no significant alterations of inflammatory cytokines (i.e. interleukin-6, TNF- $\alpha$, etc) were

Table 1 Body composition parameters at baseline and after 4, 6 and 12-months training period

\begin{tabular}{lrlll}
\hline & \multicolumn{3}{c}{ Obese women $(n=28)$} & \\
\cline { 3 - 4 } & \multicolumn{1}{c}{ T0 } & T4 & T6 & T12 \\
\hline Weight $(\mathrm{Kg})$ & $101.3 \pm 3.9$ & $94.7 \pm 3.6^{* * *}$ & $94.0 \pm 5.5^{* *}$ & $91.0 \pm 9.5^{*}$ \\
BMI $\left(\mathrm{kg} / \mathrm{m}^{2}\right)$ & $38.2 \pm 1.2$ & $35.7 \pm 1.1^{* *}$ & $35.4 \pm 1.6^{* *}$ & $34.3 \pm 3.0^{*}$ \\
FFM $(\mathrm{Kg})$ & $56.2 \pm 2.2$ & $54.9 \pm 2.2^{* *}$ & $53.8 \pm 3.3^{*}$ & $52.0 \pm 5.7^{*}$ \\
FFM (\%) & $55.5 \pm 6.5$ & $58.0 \pm 5.9^{* *}$ & $57.2 \pm 5.6^{*}$ & $57.1 \pm 5.6^{*}$ \\
FM $(\%)$ & $44.5 \pm 1.1$ & $42.0 \pm 1.3^{* *}$ & $42.8 \pm 1.6^{*}$ & $40.9 \pm 2.6^{* *}$ \\
\hline
\end{tabular}

$B M I$ body mass index, FFM fat free mass, $F M$ fat mass

$* * p \leq 0.001$ vs. T0, $* p \leq 0.05$ vs. T0 found, thus no modulation was depicted upon physical activity and nutritional intervention protocol (data not shown). As expected, physical activity protocol and nutritional intervention induced a time-dependent decrease of insulin levels, without reaching statistical significance in this population (T0: $670.23 \pm 108.88$, T4: $428.64 \pm 53.87$, T6: $418.37 \pm 86.04, \mathrm{~T} 12: 327.47 \pm 92.61 \mathrm{pg} / \mathrm{ml})$. In addition, leptin levels appeared to decrease upon treatments, without reaching significant differences (data not shown).

\section{Wnt/ $\beta$-catenin signaling in osteoblastic cells treated with trained obese patients}

Since our previous in vitro results [24] showed alteration of $\beta$-catenin translocation induced by exposure to the sera of obese subjects, first experiments were performed to characterize intracellular signaling in osteoblasts exposed to the sera of sedentary obese individuals at baseline and after a physical training period. To this aim, SAOS-2 osteoblastic cells were exposed for $48 \mathrm{~h}$ to sera of subjects affected by abdominal obesity before (T0) and after 4 (T4), 6 (T6) and 12 (T12) months of hypocaloric diet and physical activity protocol.

Exposure of osteoblasts to sera of women subjected to physical activity, induced a time-dependent total $\beta$-catenin increase already after 4 months of exercise and diet (T4) compared to the cells treated with the sera of sedentary obese individuals before the beginning of the protocol (T0) (Fig. 1a). This positive effect progressively increased during the physical activity-training period with a maximal effect reached after 12 months (T12). Accordingly to the increase
Fig. 1 Analysis of total protein content (a) and cytoplasmic and nuclear fractions (b) of $\beta$-catenin in $\mathrm{SaOS}-2$ cells cultured in the presence of obese women sera before (T0) and after 4, 6, and 12 months of physical activity protocol (T4, T6, and T12, respectively). Total, cytoplasmic and nuclear fractions of SAOS-2 cells were separated as described in "Material and methods" section. $\beta$-tubulin and histone $\mathrm{H} 3$ were used as loading and purity controls of each cellular fraction. Results are presented as mean $\pm \mathrm{SD}(n=3)$ of three separate experiments. $* * * p<0.001$, ** $p<0.01$ and $* p<0.05$ vs. T0-; \# $p<0.05$ vs. T4- and $\S p<0.05$ vs. T6treated cells
A
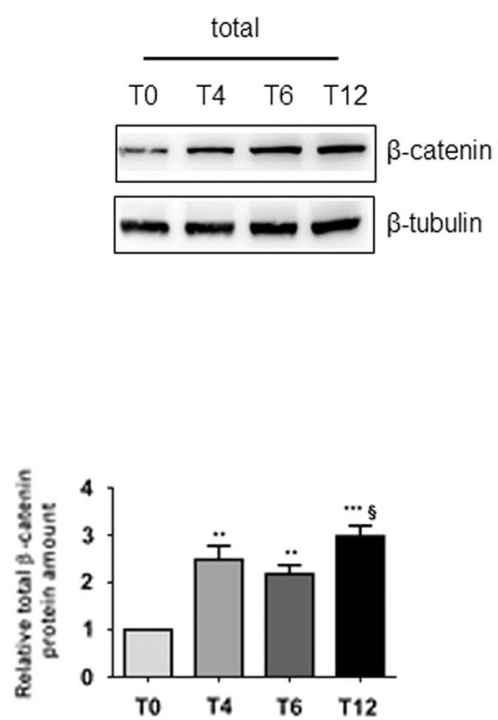

B
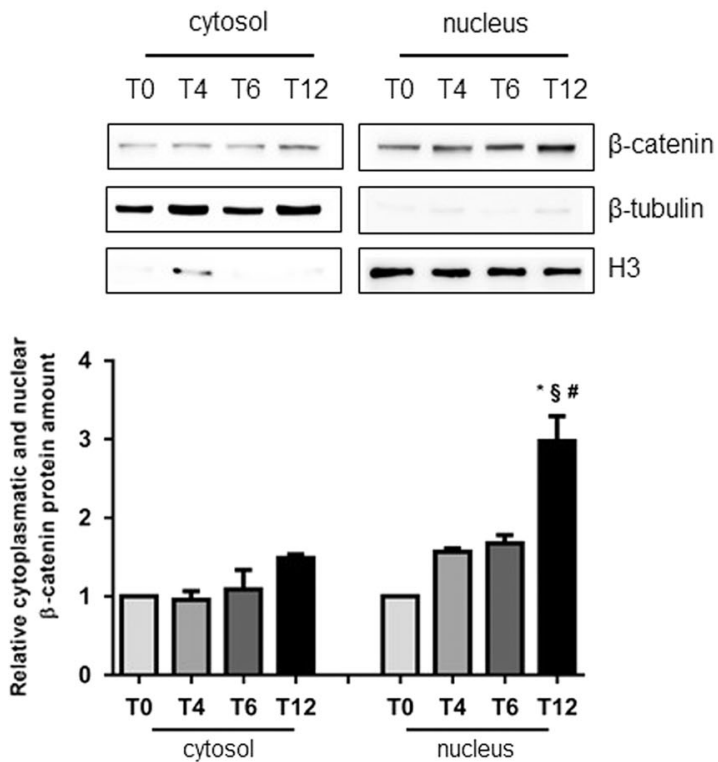
A

T0 $\quad$ T4 $\quad$ T6 $\quad$ T12
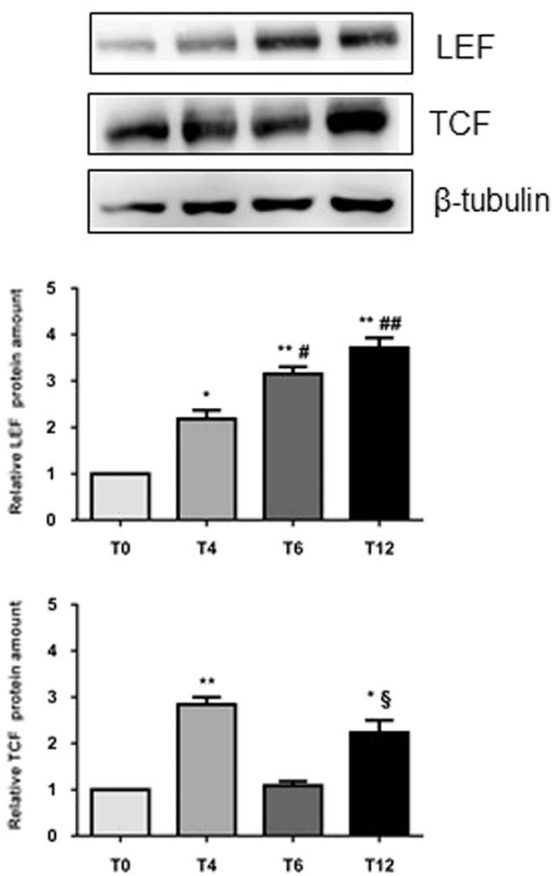

B

T0 $\quad$ T4 $\quad$ T6 $\quad$ T12

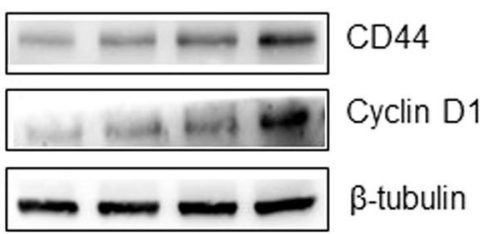

C T0 $\quad$ T4 $\quad$ T6 $\quad$ T12
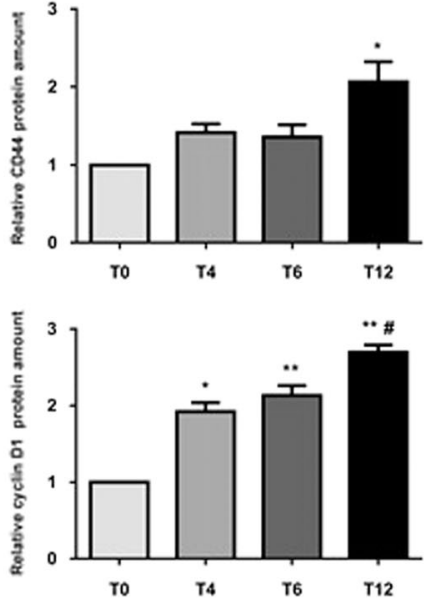
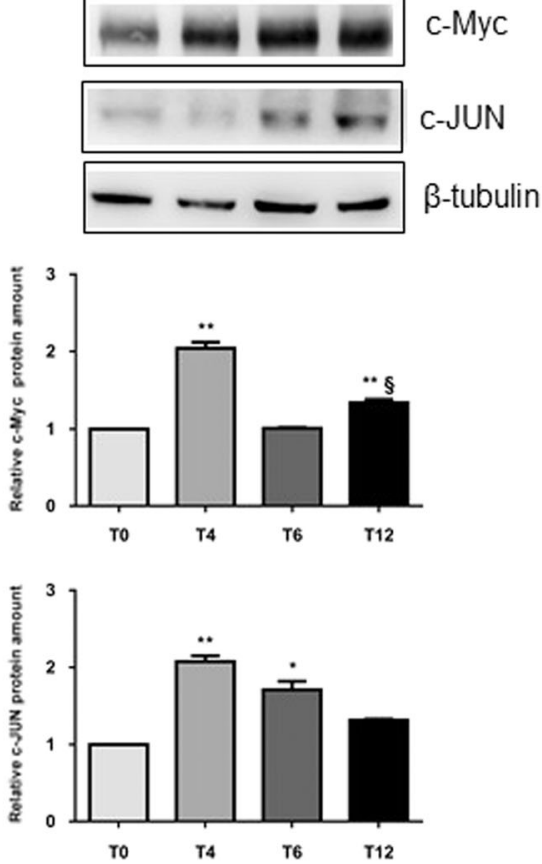

Fig. 2 Protein expression analysis of cofactors LEF/TCF (a) and specific target gene of Wnt/ $\beta$ catenin pathway, CD44, Cyclin D1 (b) and c-Myc, c-Jun (c) in SAOS-2 cells treated with sera of obese women before and after physical activity protocol. $\beta$-tubulin was used as internal control for protein loading. Results are presented as mean \pm SD $(n=3)$ of three separate experiments. $* * p<0.01$ and $* p<0.05$ vs. T0-treated cells; \# $p<0.05$, \#\# $p<0.01$ vs. T4- and $\S p<0.05$ vs. T6-treated cells of $\beta$-catenin total protein, there was also an increase of nuclear accumulation of this protein in cells exposed to T12 sera (Fig. 1b). In order to investigate the sera effects on Wnt/ $\beta$-catenin pathway activity, we also evaluated protein expression levels of $\mathrm{TCF} / \mathrm{LEF}$ transcription factors and relevant Wnt target genes including c-Myc, c-Jun Cyclin D1 and CD44.

As depicted in Fig. 2, there was a time-dependent increase of LEF protein expression in the cells treated with the T4, T6 and T12 women sera showing an increase in the ratio FFM/FM as compared to cells exposed to T0 sera. A significant increase was also been found in T6 and T12 treated cells when compared to T4-treated cells, while the expression of TCF increased about three times with T4 sera as compared to baseline. A return to the baseline levels was found in T6-treated cells followed a significant increase in T12-treated cells respect T0- and T6-treated cells (Fig. 2a). Moreover, protein expression of Cyclin D1 increased about two times in cells exposed to T4 maintaining high levels in T6- and T12-treated cells. An about two-fold increase was also found in T12 sera patients treated cells compared to baseline levels (Fig. 2b). The expression of both c-Myc and c-Jun, increased about two times with T4 sera compared to T0-treated cells, without specific further stimulatory effect at following time-points.
Interestingly, it has been recently demonstrated that Adiponectin and its receptor AdipoR1 play crucial roles in bone metabolism promoting osteoblastogenesis, osteoblast differentiation both in vitro an in vivo [30-32]. Thus, AdipoR1 was evaluated in order to further understand a potential role of this adipokine in the results observed. AdipoR1 protein levels increased in a time-dependent manner with a three-fold rise in T6- and T12-treated cells compared to baseline sera treated-cells (Fig. 3), suggesting a potential involvement of Adiponectin/AdipoR1 pathway in mediating improvement of osteoblast homeostasis likely by a $\beta$-catenin signaling recovery.

\section{Modulation in sclerostin secretion}

Since preclinical studies have shown that sclerostin inhibition increases bone formation [33-35], we evaluated sclerostin level in cellular supernatant obtained from osteoblastic cells treated with the sera of the obese women. A relevant decrease of sclerostin secretion was detected in SAOS-2 osteoblastic cells treated with $\mathrm{T} 4$, T6, and T12 sera as compared to T0 sera treated cells as showed in Fig. 4a.

Moreover, in the presence of T12 sera SAOS-2 cells showed a significant increased secretion of P1NP as compared to T0 sera treated cells (Fig. 4b), strongly 
demonstrating the positive effect of the change of life style on metabolic alteration and skeletal homeostasis.

\section{Discussion}

Our results demonstrate for the first time that the sera of obese sedentary women who improved their body composition, in terms of percentage of lean and adipose mass, upon a physical training period and hypocaloric diet, lead the re-establishment of osteoblast homeostasis as compared to cells treated with sera of the same obese subjects at baseline. In particular, sera of sedentary obese women after physical activity protocol and hypocaloric diet, activated the canonical $\mathrm{Wnt} / \beta$-catenin pathway inducing an up regulation of specific target genes with a consequent improvement of osteoblast differentiation and activity after 4, 6, and 12 months.

Indeed, it is known that the canonical $\mathrm{Wnt} / \beta$-catenin pathway is essential for proliferation and survival of

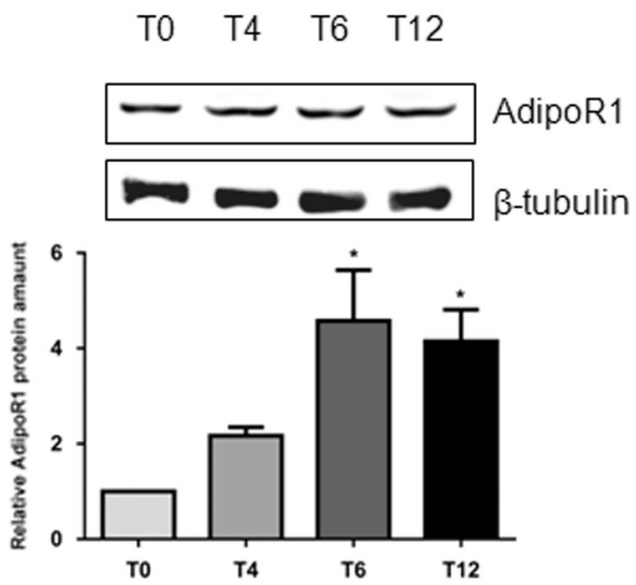

Fig. 3 Protein expression analysis of Adiponectin receptor 1 (AdipoR1) in SAOS-2 cells cultured in the presence of obese women sera before (T0) and after 4, 6, and 12 months of physical activity protocol (T4, T6, and T12, respectively). $\beta$-tubulin was used as internal control for protein loading. Results are presented as mean $\pm \mathrm{SD}(n=3)$ of three separate experiments. ${ }^{*} p<0.05$ vs. T0-treated cells osteoblasts [36]. In fact, when Wnt ligands bind to cellsurface receptors, the intracellular protein disheveled (Dvl) is phosphorylated activating a cascade of intracellular signaling. This event induces an accumulation of $\beta$-catenin in the cytoplasm and its translocation into the nucleus leading to act as transcriptional coactivator factor that belong to the TCF/LEF family linked to the modulation of target genes expression involved in cell proliferation and differentiation $[36,37]$.

Obesity and osteoporosis are two increasing health problems in many countries $[1,2]$. Obese individuals are often affected by hypertension, dyslipidaemia, diabetes mellitus and cardiovascular diseases [1, 2]; on the opposite, osteoporosis is a bone metabolic disease characterized by skeletal fragility, inducing an increased risk of developing spontaneous and traumatic fractures [6-8]. Both osteoporosis and obesity have been defined social diseases due to their high impact on mortality and morbidity, but also for the alterations in the quality of life of patients affected by these two diseases [7]. Body fat and lean mass are linked to bone mineral density, with obesity apparently exerting protection against bone loss after menopause [6-8]. The pathophysiological relevance of adipose tissue for skeletal integrity probably resides in the role of several adipokines in bone remodeling regulating both formation and resorption. For instance, serum Adiponectin level is reduced in obese subjects and increased after weight loss [38, 39] and Adiponectin /AdipoR1 pathway has received great attention due to its key role in influencing bone metabolism. Indeed, it stimulates osteoblasts proliferation and differentiation and increases bone formation [30-32], highlighting its important role as contributor to the fat-bone relationship. In fact, Lin et al. [32] indicated that AdipoR1 increases bone formation in mice and regulates osteoblasts differentiation through GSK-3 $\beta$ and $\beta$-catenin signaling. Indeed, our in vitro results indicate a recovery of $\beta$-catenin signaling, suggesting that the improved cell homeostasis observed in osteoblasts treated with the sera of trained obese women could be due to a restored activity of this transduction pathway. Moreover, the increased AdipoR1 levels further suggest a potential involvement of Adiponectin/AdipoR1
Fig. 4 Effect of obese women sera before and after diet and physical training protocol on secretion of Sclerostin (a) and $\mathrm{P} 1 \mathrm{NP}$ (b) release in culture medium of SAOS-2 cells. Results are normalized to protein content and represent mean $\pm \mathrm{SD}$ of six different wells. * $p<0.05$ vs. T0-; $\# \# p<0.01$ vs. T4- and $\S \S \S p<$ 0.001 vs. T6-treated cells
A

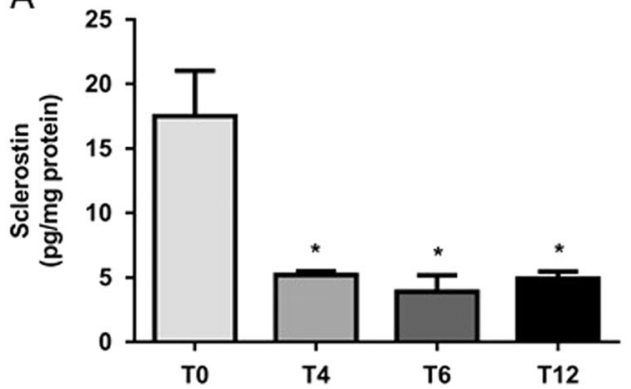

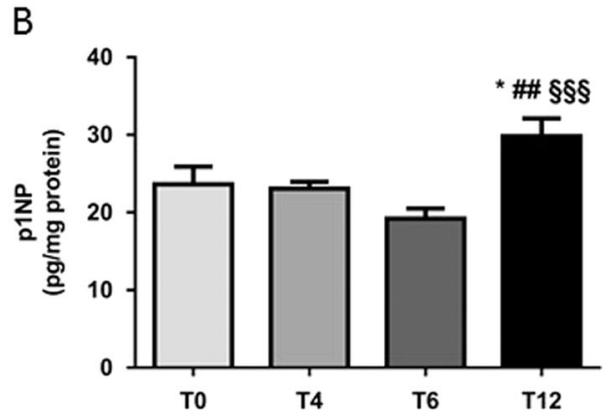


pathway in mediating $\beta$-catenin signaling recovery and improved osteoblast homeostasis.

Certainly, the cross-talk between fat and the skeleton constitutes a homeostatic feedback system in which adipokines and molecules secreted by osteoblasts represent the link of an active bone-adipose axis [21-24]. However, the mechanism(s) by which all these events occur have not been fully characterized yet. In particular, even though several data indicate that overweight women are protected from osteoporosis [8, 9], an increasing number of evidence [13] seems to show conflicting results regarding this issue, suggesting instead that obesity might actually interfere with bone health. Our previous results have shown that obese patients might indeed have disruption of skeletal metabolism, often showing an alteration of bone density or bone quality [22, 40]. Moreover, recent published data obtained in healthy premenopausal women, showed that higher abdominal adipose mass was associated with poorer bone quality, as supported by lower trabecular bone volume fraction, fewer and thinner trabeculae, lower trabecular stiffness, and higher cortical porosity [40]. In addition, the study also depicted lower trabecular bone volume fraction and decreased bone formation in vivo [40]. Indeed, our previous in vitro results supported the role of an altered bone formation in the skeletal alterations described in obese subjects [24], further emphasizing that the mechanisms underlying the observed alterations are likely due to a damaged pattern of osteoblastic differentiation by an alteration of $\mathrm{Wnt} / \beta$-catenin pathway [25].

Our previous data demonstrated that $\mathrm{Wnt} / \beta$-catenin pathway is one of the molecular intracellular pathway involved in the gene expression modulation disrupted in the osteoblasts exposed to sera of obese sedentary women [25]. Indeed, we demonstrated that GSK3 $\beta$, a key modulator of $\beta$ catenin activity, was repressed by phosphorylation at ser-9. Consequently $\beta$-catenin was slightly detectable in the cytoplasm, responsive genes were down-modulated and osteoblast differentiation and activity disrupted in the cells [25]. The results presented in this manuscript show that an improvement of body composition, likely leading to an improvement of circulating factors among which probably Adiponectin play an important role ameliorating osteoblasts differentiation and activity in vitro.

In regards of the $\mathrm{Wnt} / \beta$-catenin molecular pathway, an interesting result is linked to the evaluation and characterization of LEF1 modulation. LEF1 contains two promoters that drive expression of a full-length protein (LEF1) and a $\mathrm{N}$-terminally truncated isoform (LEF1- $\Delta \mathrm{N}$ ) [41-43]. Interestingly, while the sera of sedentary obese women blunted expression of LEF1- $\Delta \mathrm{N}$ [25], sera of the same patients, undergoing physical activity training induced a significant increase of LEF/TCF transcription factors. Moreover, specific target genes of Wnt/ $\beta$ catenin pathway, CD44, Cyclin
D1 significantly increased, further indicating that modulation of this intracellular signaling in obese subjects might play a pivotal role in the modulation of osteoblastic cells differentiation.

Indeed, physical activity seems to play an important role in maintaining skeletal health homeostasis by stimulating bone formation, through a mechanical action on osteoblasts and osteocytes [44, 45]. In addition, some recent studies seem to support a role of physical activity in the modulation of sera soluble factors, which might act on skeletal cells improving bone homeostasis [46]. Indeed the results of our study further support a role for physical activity in activating bone formation pattern by a modulation of intracellular pathways not only by a direct mechanic loading, but also by the ability of improving body composition and serum factors milieu. More interestingly, our patients were highly compliant to the physical exercise training since intervention protocol was individually planned and prescribed upon evaluation of basal physical capacity [28]. This positive effect of physical activity on body composition and, also, on osteoblastic cells homeostasis in vitro might further highlight the cellular and molecular mechanisms which positively regulate bone homeostasis as also described by others [47], further encouraging physicians and care givers to convince sedentary obese individuals to exercise even at low level of intensity.

We understand that a potential limitation of our study is linked to the fact that we have used a clonal osteoblastic cell line in vitro model system to characterize the molecular/ cellular events. However, it is well known that molecular or cellular mechanism(s) described in cellular model system are usually confirmed in primary cell lines, which we will evaluate as well.

Moreover, although it is indisputable the importance to accompany the analysis of proteins expression with those of mRNA level, the amount of sera, available for this study, was not enough to perform both analysis. However, despite this limitation, our previous published study [25] showed a tight correspondence of protein and mRNA levels in SAOS2 cells exposed to the sera of both obese patients and normal weight subjects.

In conclusion, we have described for the first time that the sera of obese sedentary women who improved their body composition, in terms of percent of lean and adipose mass, upon a physical training protocol, induce a significant improvement in osteoblast homeostasis as compared to cells treated with sera of the same obese subjects at baseline. Further studies are needed to fully characterize all events involved in the improvement of cells activity in vitro and in vivo.

Acknowledgements Research was funded by PRIN 2011052013 to SM and by PON01_00829 to AL and PRIN 2012 07/2013. V.M.B., S. F. designed the study, produced and analyzed the in vitro data, wrote 
the manuscript, contributed to the discussion; R.F. recruited and evaluated patients, contributed to the interpretation and discussion; $\mathrm{C}$. M., E.P. recruited and evaluated patients, designed dietary intervention, contributed to the interpretation and discussion. E.N. evaluated patients, performed DXA measurements, contributed to the interpretation and discussion. G.P.E., C.B., L.G. evaluated patients, trained subjects, analyzed data, contributed to the interpretation and discussion, reviewed the manuscript; L.D.L. analyzed data, contributed to interpretation, reviewed the manuscript. L.M.D. designed the study, contributed to interpretation and discussion, reviewed the manuscript; AL designed the study, reviewed the manuscript; E.A.G. and S.M. designed the study, recruited patients, analyzed the in vitro data, contributed to the interpretation, wrote the manuscript.

\section{Compliance with ethical standards}

Conflict of interest The authors declare that there is no conflict of interest that could prejudice the impartiality of the research reported.

\section{References}

1. S. Rossner, Obesity: the disease of the twenty-first century. Int. J. Obes. Relat. Metab. Disord. 26(Suppl 4), S2-S4 (2002)

2. N.I.H. Consensus, Development panel on osteoporosis prevention, diagnosis, and therapy: osteoporosis prevention, diagnosis, and therapy. JAMA. 285, 785-795 (2001)

3. D.T. Felson, Y. Zhang, M.T. Hannan, J.J. Anderson, Effects of weight and body mass index on bone mineral density in men and women: the Framingham study. J. Bone Miner. Res. 8, 567-573 (1993)

4. C. Albala, M. Yanez, E. Devoto, C. Sostin, L. Zeballos, J.L. Santos, Obesity as a protective factor for postmenopausal osteoporosis. Int. J. Obes. Relat. Metab. Disord. 20, 1027-1032 (1996)

5. P.J. Ehrlich, L.E. Lanyon, Mechanical strain and bone cell function: a review. Osteoporos. Int. 13, 688-700 (2002)

6. M.O. Premaor, L. Pilbrow, C. Tonkin, R.A. Parker, J. Compston, Obesity and fractures in postmenopausal women. J. Bone Miner. Res. 25, 292-297 (2010)

7. E.A. Greco, R. Fornari, F. Rossi, V. Santiemma, G. Prossomariti, C. Annoscia, A. Aversa, M. Brama, M. Marini, L.M. Donini, G. Spera, A. Lenzi, C. Lubrano, S. Migliaccio, Is obesity protective for osteoporosis? Evaluation of bone mineral density in individuals with high body mass index. Int. J. Clin. Pract. 64, 817-820 (2010)

8. K.C. Kim, D.H. Shin, S.Y. Lee, J.A. Im, D.C. Lee, Relation between obesity and bone mineral density and vertebral fractures in Korean postmenopausal women. Yonsei Med. J. 51, 857-863 (2010)

9. S. Cinti, The adipose organ: endocrine aspects and insights from transgenic models. Eat. Weight. Disord. 6, 4-8 (2001)

10. A.D. Attie, P.E. Scherer, Adipocyte metabolism and obesity. J. Lipid Res. 50(Suppl, ), S395-S399 (2009)

11. J. Klein, N. Perwitz, D. Kraus, M. Fasshauer, Adipose tissue as source and target for novel therapies. Trends Endocrinol. Metab. 17, 26-32 (2006)

12. J. Gomez-Ambrosi, A. Rodriguez, V. Catalan, G. Fruhbeck, The bone-adipose axis in obesity and weight loss. Obes. Surg. 18, 1134-1143 (2008)

13. J.M. Gimble, S. Zvonic, Z.E. Floyd, M. Kassem, M.E. Nuttall, Playing with bone and fat. J. Cell. Biochem. 98, 251-266 (2006)

14. E.E. Kershaw, J.S. Flier, Adipose tissue as an endocrine organ. J. Clin. Endocrinol. Metab. 89, 2548-2556 (2004)
15. P. Magni, E. Dozio, E. Galliera, M. Ruscica, M.M. Corsi, Molecular aspects of adipokine-bone interactions. Curr. Mol. Med. 10, 522-532 (2010)

16. Y. Rolland, S. Czerwinski, G. Abellan Van Kan, M. Cesari, G. Onder, J. Woo, R. Baumgartner, F. Pillard, Y. Boirie, W.M. Chumlea, B. Vellas, Sarcopenia: its assessment, etiology, pathogenesis, consequences and future perspectives. J. Nutr. Health Aging 12, 433-450 (2008)

17. A.J. Cruz-Jentoft, J.P. Baeyens, J.M. Bauer, Y. Bioirie, T. Cederholm, F. Landi, F.C. Martin, J.P. Michel, Y. Rolland, S.M. Schneider, E. Topinková, M. Vandewoude, M. Zamboni, Sarcopenia: European consensus on definition and diagnosis: report of the european working group on Sarcopenia in older people. Age Aging 39, 412-423 (2010)

18. S. Kaptoge, I.R. White, S.G. Thompson, A.M. Wood, S. Lewington, G.D. Lowe, J. Danesh, Associations of plasma fibrinogen levels with established cardiovascular disease risk factors, inflammatory markers, and other characteristics: individual participant meta-analysis of 154,211 adults in 31 prospective studies: the fibrinogen studies collaboration. Am. J. Epidemiol. 166, 867-879 (2007)

19. F.W. Kiefer, M. Zeyda, J. Todoric, J. Huber, R. Geyeregger, T. Weichhart, O. Aszmann, B. Ludvik, G.R. Silberhumer, G. Prager, T.M. Stulnig, Osteopontin expression in human and murin obesity: extensive local up-regulation in adipose tissue but minimal systemic alterations. Endocrinology 149, 1350-1357 (2008)

20. J. Wortsman, L.Y. Matsuoka, T.C. Chen, Z. Lu, M.F. Holick, Decreased bioavailability of vitamin D in obesity. Am. J. Clin. Nutr. 72, 690-693 (2000)

21. C.M. Rondinone, Adipocyte-derived hormones, cytokines, and mediators. Endocrine. 29, 81-90 (2006)

22. S. Migliaccio, E.A. Greco, R. Fornari, L.M. Donini, A. Lenzi, Is obesity in women protective against osteoporosis? Diabetes Metab. Syndr. Obes. 4, 273-282 (2011)

23. M. Zaidi, C. Buettner, L. Sun, J. Iqbal, Minireview: the link between fat and bone: does mass beget mass? Endocrinology 153, 2070-2075 (2012)

24. V. Schwetz, T. Pieber, B. Obermayer-Pietsch, The endocrine role of the skeleton: background and clinical evidence. Eur. J. Endocrinol. 166, 959-967 (2012)

25. F. Wannenes, V. Papa, E.A. Greco, R. Fornari, C. Marocco, C. Baldari, L. Di Luigi, G.P. Emerenziani, E. Poggiogalle, L. Guidetti, L.M. Donini, A. Lenzi, S. Migliaccio, Abdominal fat and sarcopenia in women significantly alter osteoblasts homeostasis in vitro by a WNT/ $\beta$-catenin dependent mechanism. Int. J. Endocrinol. 2014, 278316 (2014)

26. E.L. Rosado, R.S. de Brito, J. Bressan, J.A. Martínez Hernández, Effectiveness of prediction equations in estimating energy expenditure sample of Brazilian and Spanish women with excess body weight. Nutr. Hosp. 29, 513-518 (2014)

27. B.E. Ainsworth, D.R. Bassett Jr., S.J. Strath, A.M. Swartz, W.L. O'Brien, R.W. Thompson, D.A. Jones, C.A. Macera, C.D. Kimsey, Comparison of three methods for measuring the time spent in physical activity. Med. Sci. Sports Exerc. 32, 457-464 (2000)

28. G.P. Emerenziani, M.C. Gallotta, S. Migliaccio, E.A. Greco, C. Marocco, L. di Lazzaro, R. Fornari, A. Lenzi, C. Baldari, L. Guidetti, Differences in ventilatory threshold for exercise prescription in outpatient diabetic and sarcopenic obese subjects. Int. J. Endocrinol. 2016, 6739150 (2016)

29. G.P. Emerenziani, M.C. Gallotta, M. Meucci, L. Di Luigi, S. Migliaccio, L.M. Donini, F. Strollo, L. Guidetti, Effects of aerobic exercise based upon heart rate at aerobic threshold in obese elderly subjects with type 2 diabetes. Int. J. Endocrinol. 2015, 695297 (2015)

30. Y. Shinoda, M. Yamaguchi, N. Ogata, T. Akune, N. Kubota, T. Yamauchi, Y. Terauchi, T. Kadowaki, Y. Takeuchi, S. Fukumoto, 
T. Ikeda, K. Hoshi, U.I. Chung, K. Nakamura, H. Kawaguchi, Regulation of bone formation by adiponectin through autocrine/ paracrine and endocrine pathways. J. Cell. Biochem. 99, 196-208 (2006)

31. C.Y. Huang, C.Y. Lee, M.Y. Chen, H.C. Tsai, H.C. Hsu, C.H. Tang, Adiponectin increases BMP-2 expression in osteoblasts via AdipoR receptor signaling pathway. J. Cell. Physiol. 224, 475-483 (2010)

32. Y.Y. Lin, C.Y. Chen, T.Y. Chuang, Y. Lin, H.Y. Liu, H.J. Mersmann, S.C. Wu, S.T. Ding, Adiponectin receptor 1 regulates bone formation and osteoblast differentiation by GSK-3 $\beta / \beta$-catenin signaling in mice. Bone 64, 147-154 (2014)

33. R.D. Chapurlat, C.B. Confavreux. Novel biological markers of bone: from bone metabolism to bone physiology. (Rheumatology, Oxford), (2016)

34. X. Li, M.S. Ominsky, K.S. Warmington, S. Morony, J. Gong, J. Cao, Y. Gao, V. Shalhoub, B. Tipton, R. Haldankar, Q. Chen, A. Winters, T. Boone, Z. Geng, Q.T. Niu, H.Z. Ke, P.J. Kostenuik, W.S. Simonet, D.L. Lacey, C. Paszty, Sclerostin antibody treatment increases bone formation, bone mass, and bone strength in a rat model of postmenopausal osteoporosis. J. Bone Miner. Res. 24, 578-588 (2009)

35. M.S. Ardawi, A.A. Rouzi, M.H. Qari, Physical activity in relation to serum sclerostin, insulin-like growth factor-1, and bone turnover markers in healthy premenopausal women: a cross-sectional and a longitudinal. J. Clin. Endocrinol. Metab. 97, 3691-3699 (2012)

36. J.J. Westendorf, R.A. Kahler, T.M. Schroeder, Wnt signaling in osteoblasts and bone diseases. Gene. 341, 19-39 (2004)

37. T.P. Rao, M. Kühl, An updated overview on Wnt signaling pathways: a prelude for more. Circ. Res. 106, 1798-1806 (2010)

38. H.F. Lang, C.Y. Chou, W.H. Sheu, J.Y. Lin, Weight loss increased serum adiponectin but decreased lipid levels in obese subjects whose body mass index was lower than $30 \mathrm{~kg} / \mathrm{m}^{2}$. Nutr. Res. 31, 378-386 (2011)

39. C. Rolland, M. Hession, I. Broom, Effect of weight loss on adipokine levels in obese patients. Diabetes Metab. Syndr. Obes. 4, 315-323 (2011)
40. E. Romagnoli, C. Lubrano, V. Carnevale, D. Costantini, L. Nieddu, S. Morano, S. Migliaccio, L. Gnessi, A. Lenzi, Assessment of trabecular bone score (TBS) in overweight/obese men: effect of metabolic and anthropometric factors. Endocrine 54, 342-347 (2016)

41. R.A. Kahler, J.J. Westendorf, Lymphoid enhancer factor-1 and beta-catenin inhibit Runx2-dependent transcriptional activation of the osteocalcin promoter. J. Biol. Chem. 278, 11937-11944 (2003)

42. R.A. Kahler, M. Galindo, J. Lian, G.S. Stein, A.J. van Wijnen, J.J. Westendorf, Lymphocyte enhancer-binding factor 1 (Lef1) inhibits terminal differentiation of osteoblasts. J. Cell. Biochem. 97, 969-983 (2006)

43. L.H. Hoeppner, F.J. Secreto, D.F. Razidlo, T.J. Whitney, J.J. Westendorf, Lef1DeltaN binds beta-catenin and increases osteoblast activity and trabecular bone mass. J. Biol. Chem. 286, 10950-10959 (2011)

44. D.L. Belavý, N. Baecker, G. Armbrecht, G. Beller, J. Buehlmeier, P. Frings-Meuthen, J. Rittweger, H.J. Roth, M. Heer, D. Felsenberg, Serum sclerostin and DKK1 in relation to exercise against bone loss in experimental bed rest. J. Bone Miner. Metab. 34, 354-365 (2016)

45. G. Dallanezi, B. Funayama, A. Freire, E. Aguiar, P. Nahás, J. Nahás-Neto, J.E. Corrente, G.M. Ferreira, S. Mazeto, Physical activity level of post-menopausal women with low bone mineral density. Rev. Bras. Ginecol. Obstet. 38, 225-230 (2016)

46. J. Jürimäe, V. Tillmann, A. Cicchella, C. Stefanelli, K. Võsoberg, A.L. Tamm, T. Jürimäe, Increased sclerostin and preadipocyte factor-1 levels in prepubertal rhythmic gymnasts: associations with bone mineral density, body composition, and adipocytokine values. Osteoporos. Int. 27, 1239-1243 (2016)

47. H. Arazi, E. Eghbali, T. Saeedi, R. Moghadam, The relationship of physical activity and anthropometric and physiological characteristics to bone mineral density in postmenopausal women. J. Clin. Densitom. 19, 382-388 (2016) 